chercher : repérer : avancer

Cet article est disponible en ligne à l'adresse :

http://www.cairn.info/article.php?ID REVUE=RISS\&ID NUMPUBLIE=RISS 171\&ID ARTICLE=RISS 171_0095

Bien privé, bien collectif et bien public à l'âge de la génomique

par Maurice CASSIER

érès | Revue internationale des sciences sociales

$2002 / 1-N^{\circ} 171$

ISSN 3034-3037 | ISBN 2-7492-0042-3 | pages 95 à 110

Pour citer cet article :

- Cassier M., Bien privé, bien collectif et bien public à l'âge de la génomique, Revue internationale des sciences sociales 2002/ 1, N 171, p. 95-110.

Distribution électronique Cairn pour érès.

(C) érès. Tous droits réservés pour tous pays.

La reproduction ou représentation de cet article, notamment par photocopie, n'est autorisée que dans les limites des conditions générales d'utilisation du site ou, le cas échéant, des conditions générales de la licence souscrite par votre établissement. Toute autre reproduction ou représentation, en tout ou partie, sous quelque forme et de quelque manière que ce soit, est interdite sauf accord préalable et écrit de l'éditeur, en dehors des cas prévus par la législation en vigueur en France. Il est précisé que son stockage dans une base de données est également interdit. 


\title{
Bien privé, bien collectif et bien public à l'âge de la génomique
}

\author{
Maurice Cassier
}

\section{Introduction}

Dans son ouvrage sur « le commerce des gènes dans le meilleur des mondes », J. Rifkin (1998) établit un parallèle entre l'extension contemporaine des droits de propriété intellectuelle sur les ressources génétiques et la loi sur les enclosures, qui organisa au XVII ${ }^{\mathrm{e}}$ siècle la privatisation des biens communaux en Angleterre 1. Cette tendance à la privatisation renvoie à une intégration inédite de la science et des marchés ${ }^{2}$. Premièrement, il s'est formé au cours des années quatre-vingt-dix un secteur et un marché privés de la recherche génomique, avec la création de sociétés de recherche directement adossées aux marchés financiers. Deuxièmement, les agences publiques de recherche et les institutions académiques ont intensifié la commercialisation de leurs travaux, encouragées par l'adoption de lois qui
Maurice Cassier est sociologue au CNRS à Paris. Il travaille au Centre de recherche médecine, sciences, santé et société. Ses travaux portent sur les relations entre l'université et l'industrie, sur l'intégration de la science, de la médecine et du marché dans le domaine de la génomique et sur la confrontation entre la propriété industrielle et la santé publique, aujourd'hui et au XIX ${ }^{\mathrm{e}}$ siècle. Email : cassier@ext.jussieu.fr. des ressources et des connaissances sur les génomes, est battu en brèche par le « modèle de la privatisation ». Au point qu'un excès de droits privatifs accumulés et superposés sur le génome humain pourrait provoquer une « tragédie des anticommuns »(Heller et Eisenberg, op. cit.), situation dans laquelle il devient trop coûteux pour les innovateurs de réunir et de lever les multiples droits nécessaires au développement des innovations médicales.

Cette tendance à la privatisation de la recherche, bien que très forte dans le domaine du génome humain, n'est toutefois ni unilatérale, ni complètement stabilisée. Il existe en effet dans le domaine de la génétique une tradition de recherche collective, particulièrement pour la cartographie des gènes. Dès les années trente, le généticien américain Morgan construisait un réseau de laboratoires qui coopéraient pour élaborer la cartographie visaient à augmenter l'appropriabilité et la transférabilité des connaissances produites sur fonds publics. Cette intégration nouvelle de la recherche et des marchés débouche sur le brevetage massif des séquences génétiques, l'extension de bases de données protégées par le secret commercial, l'établissement de contrats d'accès exclusif aux données génétiques et médicales de certaines populations, la multiplication d'accords de recherche ou de transfert de matériels qui définissent des droits d'usage réservé. Le « modèle du commun » (Heller et Eisenberg, 1998), fondé sur la libre accessibilité et la large dissémination génétique de la drosophile. Dans ce réseau d'une demi-douzaine de laboratoires, les mutants de drosophiles nouvellement identifiés étaient mis à la disposition des autres équipes dans un contexte de réciprocité (Kholer, 1994). Une autre initiative marquante fut celle du réseau constitué par le Centre d'Étude du Polymorphisme Humain (CEPH) pour cartographier le génome humain au début des années quatre-vingt, à l'initiative de Jean Dausset et de Daniel Cohen. Le CEPH, qui est une fondation de recherche créée avec des fonds d'une donation privée, constitua un pool de familles de référence, d'origines française et 
américaine, qui fut mis à la disposition des laboratoires intéressés par la construction d'une carte génétique humaine. L'usage commun de familles de référence favorisant l'avancée cumulative des connaissances, les résultats étant stockés dans une base de données centrale accessible à tous les participants. Cette recherche collective était davantage formalisée que les réseaux de collaboration de Morgan ou de Delbrück 3 dans les années trente ou quarante. Pour accéder à la collection d'ADN du CEPH, les laboratoires devaient adhérer aux principes d'échange de données qui avaient été fixées. On retrouve ce modèle de recherche collective, diversement accompli, dans les consortiums européens financés par les programmes de recherche de la Communauté économique européenne (CEE) sur les biotechnologies, à partir de la fin des années quatre-vingt. Le séquençage du génome de la levure, puis les débuts de l'étude fonctionnelle des gènes de la levure, supportèrent la création de réseaux de laboratoires à l'échelle européenne, unis par des principes de division du travail, d'échanges de données et de répartition de la propriété des résultats. Fait plus remarquable encore, les firmes industrielles sont susceptibles d'organiser des consortiums de recherche qui produisent des biens publics, à l'image du consortium sur les Single Nucleotide Polymorphisms (SNPs) créé en juillet 1999 à l'initiative des dix plus grands laboratoires pharmaceutiques mondiaux. Ces organisations de recherche collective, auto-organisées par les scientifiques ou créées à l'initiative des États ou des firmes, viennent disputer au secteur privé le contrôle des connaissances - la concurrence entre le consortium public pour le séquençage du génome humain et la société privée Celera Genomics - ou apporter des corrections ou des solutions à l'excès de droits privés qui prolifèrent sur les génomes - par exemple, les formules de propriété collective des connaissances conçues par certains consortiums européens ou l'initiative du laboratoire Merck en 1994 de diffuser des étiquettes de gènes dans le domaine public. Il existe également de nombreuses propositions visant à limiter le droit de la propriété intellectuelle dans le domaine de la génomique, par le jeu d'exclusions de la brevetabilité, de licences obligatoires ou volontaires dans le domaine des usages pour la santé, ou encore de systèmes de droits organisant des pools de connaissances libres.
Cet article vise à présenter à la fois l'étendue et la variété des pratiques d'appropriation privative de la recherche génomique, ainsi que, en contrepoint ou en contre-tendance, les différentes organisations et conventions qui définissent des espaces collectifs et/ou publics de recherche. La première section étudiera l'extension des droits exclusifs sur les génomes. La seconde section analysera les pratiques de brevetage des gènes humains et leurs effets sur l'offre de santé d'une part, sur la recherche biomédicale d'autre part. La troisième section exposera différents modèles de production et de distribution de biens collectifs et/ou de biens publics. La conclusion reviendra sur des propositions visant à réorienter le système des brevets dans le domaine du génome ou à envisager d'autres modèles de gestion des ressources et des connaissances génomiques en termes de bien commun pour la recherche et la santé publique.

\section{La tendance à l'extension de droits exclusifs sur la recherche génomique}

Si les brevets constituent le cœur de l'appropriation privative de la recherche génomique, les sociétés de biotechnologie comme Genset ou Myriad Genetics utilisent parallèlement aux brevets plusieurs autres outils d'appropriation pour protéger des ressources ou des connaissances non brevetables en l'état - collections de données généalogiques ou médicales utilisées comme ressources pour l'identification des gènes, données intermédiaires de recherche pour la localisation et la caractérisation des gènes, etc. ${ }^{4}$. On étudiera successivement :

Les contrats d'usage exclusif des collections de données génétiques et médicales ;

L'extension des bases de données privées protégées par le secret;

L'extension du champ de la brevetabilité aux séquences génétiques.

\section{Les contrats d'accès exclusifs aux ressources génétiques et médicales des populations humaines}

L'appropriation de la recherche génomique commence avec le contrôle des bases de données médicales et génétiques qui permettent de localiser des gènes ou d'envisager des essais thérapeu- 
tiques fondés sur les variations génétiques des populations. L'accès à des données bien documentées ou à des groupes de population très spécifiques peut représenter une véritable rente d'innovation pour les acteurs de la génomique médicale. La ressource en population explique parfois la localisation géographique de certains chercheurs ou de certaines sociétés de génomique. Les fiches de présentation au marché boursier de la société américaine Myriad Genetics font valoir l'usage exclusif que détient la société sur plusieurs bases de données familiales et médicales : «Un des facteurs clés de la réussite de Myriad Genetics a été le développement et l'exploitation de bases de données génétiques et médicales privatives. Notre société détient des droits d'accès exclusifs à des bases de données généalogiques de familles de l'Utah et de familles de Canadiens français ${ }^{5}$. » En France, la société Genset s'efforce également d'établir des contrats d'accès exclusif à des collections de données cliniques.

Jusqu'ici, ces données étaient échangées dans des réseaux académiques, sur la base d'accords tacites, entre un chercheur et un clinicien, ou dans le cadre d'échanges formalisés, comme à l'intérieur du réseau constitué par le Centre d'étude sur le polymorphisme humain dans les années quatre-vingt. La privatisation de ces données n'est pas seulement le fait de contrats privés passés entre des firmes et des institutions médicales. Les licences exclusives attribuées par des États à des sociétés de biotechnologie pour exploiter les données génétiques et médicales de certaines populations, en Islande ou aux îles Tonga par exemple, aboutissent également à la mise sur le marché de ces données.

En Islande, le processus de valorisation et d'appropriation des données relatives à la population commence en 1996, lorsque le fondateur de DeCode réunit des fonds de capital-risque aux États-Unis, pour un montant de 12 millions de dollars, en faisant valoir dans son business plan la spécificité génétique de la population islandaise - son homogénéité génétique supposée - et l'excellence des enregistrements des données médicales réalisés par le système national de santé. La valeur d'usage potentielle de ces données permit à DeCode de conclure un très gros accord de recherche avec Hoffman Laroche. Ce contrat, signé en février 1998 pour une durée de cinq années, prévoit que DeCode utilisera la base de données sur la population islandaise pour isoler des gènes pathogènes qu'elle fournira à Roche, à charge pour celle-ci de concevoir de nouveaux médicaments ou de nouveaux outils diagnostiques - Roche recevant un droit exclusif pour les utilisations thérapeutiques et diagnostiques des gènes intéressant douze maladies. En contrepartie, elle apporte à DeCode des fonds en capital, finance des opérations de recherche et verse des royalties en cas de mise sur le marché de produits. Il est également stipulé un retour vers la population : en cas de développement de produits issus de la collaboration, Roche les fournira gratuitement aux Islandais. Le troisième élément de cette construction entre la population islandaise, une société privée de recherche et le laboratoire pharmaceutique Roche est le vote d'une loi par le parlement islandais qui autorise la concession d'une base de données médicales nationale à un acteur privé, en l'occurrence DeCode Genetics. Après l'adoption de cette loi, en décembre 1998, par trente-huit voix contre vingt-trois, la capitalisation boursière de DeCode est multipliée par quatre. La licence sera concédée à DeCode en janvier 2000 pour une durée de douze années. Cette chronologie montre que les marchés et l'attribution de droits exclusifs sont au cœur de la recherche génomique. Au terme de ce processus, la population islandaise, son profil génétique et ses informations médicales sont le support de la valorisation de cette société privée qui se présente comme une «société fondée sur la population ${ }^{6} \gg$.

La loi islandaise confère aux collections de données génétiques et médicales d'une population certains attributs d'un bien privé. En contrepartie du paiement d'un droit d'accès, une société peut acquérir un droit exclusif pour l'exploitation de ces collections, ce qui s'apparente à l'octroi d'une concession minière. L'exclusivité de la concession génère une rente pour le licencié, d'autant plus forte que le profil génétique de la population est spécifique et que les données médicales enregistrées sont de bonne qualité. Le caractère de propriété privée de ces collections de données est toutefois tempéré par certaines dispositions de la loi, qui limitent la transférabilité de la base de données. Celle-ci doit être localisée en Islande et ne peut être engagée dans des opérations financières - le licencié ne peut ni l'hypothéquer ni revendre la licence. Le gouvernement a un droit d'accès à la base de données pour effectuer des études statistiques pour la santé publique. 
L'attribution de cette concession est ainsi justifiée par le gouvernement :

Si le gouvernement reconnaît que les données médicales enregistrées par le système de santé sont une "ressource nationale... qui ne peut être un objet de propriété au sens habituel du terme », il justifie l'intervention d'un acteur privé par le montant de l'investissement nécessaire à la construction d'une base de données nationale ;

La population et le système de santé bénéficieront de l'acquisition de nouvelles connaissances, éventuellement de nouveaux produits de santé ;

Cette base de données aura des retombées économiques positives en termes de création d'une industrie biotechnologique locale.

L'attribution d'un droit d'accès exclusif aux données génétiques et médicales d'une population a provoqué une vive controverse. L'accès réservé aux données, s'il augmente leur valeur commerciale, limite la variété des investigations de recherche possibles, ce qui peut in fine freiner les avancées scientifiques et médicales ${ }^{7}$. Des chercheurs dénoncent le «monopole » de DeCode sur les données islandaises au nom de " la science ouverte ». A contrario, la base de données du Royaume-Uni, The UK Population Biomedical Collection, constituée à l'initiative du Medical Research Council et de la Wellcome Foundation et qui devrait porter sur cinq cent mille individus, sera accessible aux firmes privées sur une base non exclusive. Le second point de controverse porte sur l'étendue du consentement des personnes à participer à cette base de données et à se prononcer sur les usages qui pourront en être faits. La notion de « consentement présumé », qui confère aux personnes un simple droit de se retirer de la base de données médicales ${ }^{8}$ et qui a été étendue aux échantillons d'ADN par le Biobank Act en mai 2000, est très discutée. Des médecins ont décidé de ne pas adresser d'information à la base de données sans demande explicite des patients.

\section{Les bases de données génomiques protégées par le secret commercial et vendues par contrats}

L'extension du secteur privé de la recherche s'est accompagnée de la multiplication des bases de données privées sur les génomes, humains et non humains. Ainsi, en 1995, un rapport de l'OCDE sur le grand programme sur le génome humain note que la majeure partie des séquences partielles sont retenues dans des bases de données privées : "Quelques-uns de ces marqueurs exprimés de séquence (MES) sont dans le domaine public, notamment ceux générés en France et au Royaume-Uni, mais le travail s'est largement effectué dans le secteur privé ; il en résulte que la plupart de ses séquences sont gardées secrètes. L'OCDE souligne les risques de redondance croissante entre ces diverses collections confidentielles et « les possibilités de disputes sur la priorité et le droit de propriété ». L'OCDE relève toutefois l'initiative de Merck, lancée en 1994, visant à produire une collection de séquences partielles destinée à être placée dans le domaine public (Eisenberg, 1996).

L'opposition entre le secteur public et le secteur privé de la recherche a été singulièrement forte lors du séquençage massif du génome humain, à la fin des années quatre-vingt-dix. Tandis que le consortium du Human Genome Project (HGP), soutenu par des agences publiques de recherche et une fondation privée, la Wellcome Foundation, se mettait en place pour coordonner le séquençage des chromosomes humains et mettre la séquence dans le domaine public, Craig Venter, qui faisait initialement partie du consortium, annonça en mai 1998 la création de Celera Genomics pour développer un projet commercial concurrent. Le secteur public réagit par deux plans successifs d'accélération de son programme. L'investissement massif de Celera visait à prendre de vitesse la production et la publication des données du consortium international. En attendant de pouvoir disposer d'éventuels brevets sur des gènes d'intérêt médical, le monopole de la société privée reposait sur une fenêtre étroite, celle que lui conférerait son avance sur le consortium public. Toutefois la valeur commerciale des données de Celera reposait aussi sur une certaine spécificité - l'inclusion de certains polymorphismes - et sur l'addition de ses données propres à celles du consortium public. En effet, au cours de cette course de vitesse, l'acteur privé bénéficiait d'une asymétrie d'information vis-àvis de l'acteur public. Tandis que les chercheurs du consortium public ne pouvaient accéder aux données produites par Celera, cette dernière intégrait les données publiques dans sa base de données, ce qui lui conférait un avantage en termes d'information qui était susceptible d'intéresser les souscripteurs. Cette asymétrie joua également 
au moment de la négociation avec le consortium public pour la fusion de leurs données : quoi qu'il advienne, Celera continuerait à bénéficier de la politique de diffusion immédiate du HGP.

Le délai de divulgation des données fut au centre de la controverse engagée entre le consortium public et Celera à la fin de l'année 1999, dès lors qu'il fut question de fusionner les séquences produites par les deux acteurs pour accélérer le programme de séquençage. Afin de préserver son intérêt commercial, Celera demandait un délai de trois à cinq ans pour la diffusion des données issues de la collaboration avec le HGP, qu'elle assemblerait dans ses murs et qui seraient distribuées à l'industrie. La discussion achoppa sur le délai de libre accès des séquences : le HGP ne lui consentait que six à douze mois de réservation des données.

La publication de la séquence produite par Celera en février 2001 dans Science, en parallèle de la publication de la séquence du HGP dans Nature, s'est accompagnée de certaines restrictions. Si la séquence complète de Celera est librement accessible pour les chercheurs académiques, le chargement de morceaux de séquences supérieurs à un mégabase nécessitera l'établissement d'un accord avec l'institution de recherche qui l'oblige à ne pas redistribuer la séquence. Des chercheurs en bio-informatique se sont élevés contre une telle restriction dans la mesure où ils travaillent sur de grands ensembles de séquence. Des scientifiques pensent que ces conditions d'accès sont trop compliquées et qu'elles vont dissuader les utilisateurs. Quant aux utilisateurs industriels qui voudront accéder aux données, ils devront signer un accord de transfert de matériel qui leur impose de ne pas commercialiser ou redistribuer les données. A moins qu'ils ne souscrivent à la base de données ou ne concluent un accord de licence. Le président de l'Académie des sciences des États-Unis a demandé que les données soient accessibles à la fois pour le secteur public et le secteur privé. Cet accord conduit à une détérioration des normes de publication : il instaure un précédent pour les scientifiques qui pourraient désormais publier sans être obligés de ménager un accès aux données complètes qui supportent la publication ; il rompt l'unicité des dépôts dans les banques de données publiques en autorisant le dépôt des données qui sous-tendent la publication sur le site propre du déposant.

La publication d'une première version de la séquence brute du génome humain, par Celera et le HGP, ne ruine pas toute possibilité de commercialisation des données génomiques. La commercialisation se déplace simplement vers les connaissances sur la fonction biologique des gènes, sur les protéines ou des marqueurs originaux.

La valeur commerciale des bases de données ne repose pas seulement sur le secret ou le délai d'avance. Elle rémunère également l'originalité et la spécificité des données produites, eu égard aux méthodes de recherche et aux traitements bio-informatiques employés. Par exemple, Genset a construit une base de données sur un sousgroupe d'étiquettes de gènes qui repèrent les extrémités de gènes de protéines sécrétées. Ces gènes sont détectés grâce à un procédé bio-informatique original. De la même manière, Myriad Genetics a développé une base de données sur les interactions de protéines qu'elle exploite à travers plusieurs contrats de recherche passés avec des laboratoires pharmaceutiques. La spécificité des traitements bio-informatiques est un élément clé de l'appropriabilité et de la valorisation des bases de données. Outre le savoir-faire bio-informatique de l'entreprise, la valeur commerciale des bases de données tient également à la spécificité des ressources génétiques et médicales collectées, à l'image des collections de données exploitées par DeCode ou Myriad Genetics. De telles bases de connaissances deviennent des points de passage obligés pour les laboratoires pharmaceutiques.

On peut distinguer plusieurs stratégies de commercialisation des bases de données. On peut opposer la politique de licence exclusive de la société Human Genome Science (HGS) à la politique de licences non exclusives d'Incyte. HGS a choisi de valoriser sa base de données en concluant un gros accord de recherche avec SmithKline and Beecham en 1993, qui donnait à cette dernière un droit d'usage exclusif sur les diagnostics, les vaccins, les produits thérapeutiques obtenus à partir de sa base - HGS gardant des droits sur la thérapie génique et les antisens. Plus récemment, HGS et SB ont fait évolué l'accord pour permettre à d'autres firmes pharmaceutiques d'accéder à cette base de données. Incyte a adopté d'emblée une politique de licences non exclusives, qui a attiré les laboratoires qui n'avaient pu accéder à la base de données de HGS. Si bien qu'elle vend des licences à dix-huit des vingt premiers laboratoires pharmaceutiques mondiaux. Le président d'Incyte justifie ainsi sa 
politique de licences non-exclusives : les multiples laboratoires pharmaceutiques souscripteurs partagent les coûts de recherche de sa société, ce qui lui permet de développer plus rapidement sa base de données. Si la base de connaissances d'Incyte devient suffisamment large, les firmes pharmaceutiques n'auront pas à négocier des licences auprès de multiples propriétaires de brevets.

Myriad Genetics découpe quant à elle des exclusivités par pathologie, soit des field exclusive agreements, et propose aux firmes pharmaceutiques un partage des droits de propriété, entre les usages thérapeutiques, qu'elle cède, et les usages diagnostiques des gènes brevetés, qu'elle se réserve pour commercialiser directement des tests génétiques. Plus récemment, Myriad a négocié avec les laboratoires pharmaceutiques des formules de codéveloppement des produits thérapeutiques impliquant un partage des bénéfices des ventes de médicaments sur le marché des États-Unis. Le partage de la propriété évolue au fur et à mesure que les sociétés de génomique ne se limitent plus à la vente de l'information génétique, mais intègrent le prédéveloppement de molécules.

\section{La mobilisation du droit des brevets pour protéger la recherche génomique}

Les récentes guidelines publiées par l'USPTO sur l'utilité 9 pour servir de guide aux examinateurs de brevets dans le domaine des inventions génétiques ont l'intérêt de présenter les justifications de la brevetabilité des séquences génétiques. Il n'existe pas de règle a priori permettant de rattacher les nouveaux objets qui émergent à tel ou tel droit de propriété intellectuelle ${ }^{10}$. Dans le domaine de la génomique, ce sont principalement les professionnels de la propriété industrielle - conseils en propriété industrielle, examinateurs des offices de brevets - ainsi que les industriels qui ont « fait » le droit dans ce domaine ${ }^{11}$.

La construction de ce cadre d'appropriation repose sur quelques interprétations clés :

La première opération a consisté à rattacher la génomique au génie chimique en établissant une équivalence entre les molécules d'ADN et les molécules chimiques. Cette équivalence est exprimée par les spécialistes de la propriété industrielle (« Je ne suis pas d'ac- cord avec vous. Une séquence d'ADN, ce n'est pas de l'information, mais une formule chimique d'un produit »- conseil en brevet), ou par des industriels ( «Si vous découvrez une nouvelle molécule, qu'il s'agisse d'une matière plastique, d'un médicament, d'une peinture, d'un colorant ou d'un gène, c'est bien une nouvelle molécule; vous devez la protéger et les brevets sur les gènes suivent le modèle qui a été mis en place pour la chimie organique »-Mark Skolnick, fondateur de Myriad Genetics). L'USPTO réaffirme la même doctrine : "Si les brevets sur les gènes sont traités de la même manière que pour les autres composés chimiques, cela stimulera le progrès parce que l'inventeur originel aura la possibilité de récupérer ses frais de recherche, parce que les autres inventeurs seront stimulés pour inventer autour du premier brevet, et parce qu'un nouveau composé chimique sera rendu accessible pour de futures recherches »;

La seconde opération a consisté à étendre la notion d'invention à tout travail d'isolement des choses naturelles que l'on décrit, manipule, isole, reproduit ${ }^{12}$. Les concepts clés pour définir l'invention étant désormais l'intervention humaine sur la nature et l'utilité : « C'est là où finalement intervient l'inventeur, c'est-à-dire l'intervention de l'homme sur la nature, c'est-à-dire avoir extrait cette séquence de son environnement plus complexe pour la mettre dans un environnement différent, l'ADN recombinant, pour permettre l'expression d'une protéine qui aurait un intérêt biologique, pharmaceutique ou agroalimentaire ou végétal » (conseil en brevets) ;

La troisième opération a consisté à préciser la notion d'utilité ou d'application industrielle appliquée aux brevets sur les séquences génétiques. Les controverses ont porté sur la brevetabilité des séquences partielles de gènes. En 1991, la demande des NIH de breveter des séquences fragmentaires d'ADN suscita une forte opposition (Cook-Degan, 1995). Le brevet des NIH - dont Craig Venter était l'inventeur- portait sur plusieurs centaines d'étiquettes de gènes qui permettaient d'identifier un gène exprimé. Bien que les fonctions des gènes correspondants ne fussent pas établies, les NIH justifiaient leur demande de brevet par le fait que ces éti- 
quettes étaient des indices pour découvrir de futurs gènes. Ils craignaient également que la publication de ces séquences fragmentaires rende impossible le brevetage des gènes complets qui seraient isolés par la suite. Les industriels étaient partagés sur la validité et l'utilité de tels brevets. Si The Industrial Biotechnology Association se prononçait pour le dépôt de ces brevets, elle demandait que les NIH mettent dans le domaine public les brevets qui portent sur des séquences partielles de gènes - seuls les brevets décrivant le gène complet et sa fonction biologique devant être exploités. Finalement, l'USPTO rejeta la demande au motif que celle-ci ne répondait pas au critère d'utilité. Toutefois, l'attribution par l'USPTO d'un brevet sur quarante-quatre étiquettes de gènes à la société Incyte en 1999 montre que la question n'est pas résolue. La portée des revendications de ce brevet est singulièrement large : comme pour le brevet des NIH en 1991, les revendications portent non seulement sur l'utilisation des étiquettes comme sonde, mais également sur les gènes entiers qu'elles indiquent et sur les protéines correspondantes. L'application des guidelines sur l'utilité devrait permettre d'éliminer les demandes de brevet les plus spéculatives, dès lors qu'une utilité spécifique, « pour un usage pratique particulier », n'est pas justifiée ;

Le quatrième point controversé est celui de l'interprétation du critère d'activité. Sur ce point aussi, le brevet des NIH de 1991 suscita une forte opposition : la Human Genome Organization (HUGO) s'opposa fermement au brevetage des séquences partielles d'ADN au motif que le décryptage de la séquence n'était pas une activité inventive. Pire, en attribuant des droits de propriété sur des séquences dont la fonction biologique et l'utilité n'étaient pas connues, le système des brevets récompensait l'activité routinière au détriment du travail créatif ${ }^{13}$. HUGO souhaitait une réorientation du système des brevets : c'est le travail d'interprétation des gènes qui devait être récompensé par des brevets, non le travail de décryptage. Tout en encourageant la divulgation des séquences dans le domaine public, HUGO entendait préserver les possibilités de protection industrielle des gènes d'intérêt médical.

\section{La structure des brevets sur les gènes et la recherche biomédicale et l'offre de soins}

En décembre 1999, le Secretary Advisory Committe on Genetic Testing, mis en place auprès du département d'État à la Santé pour faire des recommandations sur la régulation du marché des tests génétiques aux États-Unis, faisait le constat suivant : «Des craintes se sont récemment élevées concernant l'impact du brevetage des gènes humains sur les activités de test génétique... Alors qu'en règle générale les brevets apportent une incitation à développer des applications utiles, certains détenteurs de brevets ont commencé à restreindre l'usage des gènes qu'ils ont découverts en imposant des prix très élevés pour l'attribution de licences, en imposant des licences exclusives, ou en refusant purement et simplement de concéder des licences. Ces restrictions peuvent avoir des effets négatifs sur l'accessibilité, le prix et la qualité des tests génétiques » $(A$ Public Consultation on Oversight of Genetic Tests, 1999). On analysera ici la structure particulière des brevets pris sur les séquences génétiques et leur impact sur la dynamique de recherche, d'une part, sur l'offre de soins, d'autre part.

\section{Des brevets de produit et d'applications}

Qu'ils soient déposés par des start-up de génomique, par des universités ou par des fondations de recherche médicale, et quelles que soient les motivations des déposants ${ }^{14}$, ces brevets ont des structures très similaires. Cette similitude s'explique par la circulation de modèles de rédaction des brevets dans les communautés de spécialistes de la propriété industrielle et par leur fixation progressive par les offices de brevets, le cas échéant par les juges.

Premièrement, ces brevets portent à la fois sur la séquence génétique et sur les applications qui en dérivent. Le brevet de base déposé par Myriad Genetics sur le premier gène de prédisposition du cancer du sein, qui s'intitule «17q-Linked Breast and Ovarian Cancer Susceptibility Gene », revendique à la fois « les matériaux et les méthodes » du diagnostic génétique. L'essentiel tient au fait que la séquence n'intervient pas seulement comme un élément de description de l'invention, mais qu'elle est l'objet même des revendications. Ainsi les onze premières revendi- 
cations du brevet de base de Myriad Genetics portent sur la séquence isolée du gène $-\mathrm{y}$ compris ses formes mutées. Suivent des revendications sur les usages de la séquence comme sonde ou comme amorce, puis sur la protéine codée par ce gène. Le brevet de la Cancer Research Campaign sur le second gène de susceptibilité du cancer du sein épouse la même structure : les premières revendications du brevet couvrent « une molécule d'acide nucléique comprenant la séquence codante ou le gène complet BRCA2 $»$.

Le gène et la protéine sont brevetés comme de nouvelles compositions de matière révélées et mises à disposition par l'intervention humaine. Les guidelines de l'USPTO justifient ainsi la brevetabilité des séquences génétiques : «Un gène isolé est brevetable comme composition de matière ou comme article de manufacture dans la mesure où cette molécule d'ADN n'existe pas sous cette forme isolée dans la nature. » À l'instar des brevets sur les composés chimiques, il s'agit de brevets de produit qui couvrent la molécule d'ADN et toutes les utilisations qui pourront en être faites, connues ou inconnues au moment du dépôt de brevet ${ }^{15}$. Ce faisant, ces brevets instaurent une chaîne de dépendance vis-à-vis du premier découvreur du gène : tout nouvel utilisateur doit passer par le premier propriétaire pour être autorisé à exploiter une invention nouvelle. La dépendance est d'autant plus forte dans le domaine de la recherche génomique qu'il n'existe qu'un seul génome ${ }^{16}$. Il n'existe en effet qu'un seul gène BRCA1 et un seul gène BRCA2 dans le domaine du cancer du sein. La seule limitation du pouvoir d'un tel brevet peut venir de la découverte d'un gène plus essentiel pour la maladie en question.

Ensuite, ces brevets sont généralement très larges. Tout d'abord parce que la propriété du gène ouvre le droit à une cascade de produits liés : la protéine et ses antagonistes ${ }^{17}$, ainsi que les cellules modifiées ou les animaux transgéniques porteurs du gène. Ensuite, parce que ces brevets revendiquent une large gamme d'applications, diagnostiques, thérapeutiques ou encore les méthodes de screening des médicaments. Dans le cas des brevets de Myriad Genetics sur les gènes du cancer du sein, les revendications couvrent tout usage diagnostique sans limitation de procédés. Cela signifie par exemple que toutes les méthodes de tests génétiques qui ont été développées par de nombreux laboratoires cliniques tombent sous le coup de ces brevets.
L'inclusion de la séquence des gènes dans les revendications des brevets et la définition de brevets larges ont été justifiées par le souci de donner une prime au premier découvreur et par la volonté de fonder une nouvelle industrie 18 .

\section{Les effets sur la recherche et l'innovation médicale}

L'impact de tels brevets sur la recherche biomédicale est très ambivalent. D'un côté, l'attribution d'un droit exclusif au premier découvreur crée des incitations à investir dans la recherche génomique et dans les applications médicales. De plus, le système des brevets conduit à la divulgation des connaissances sur les gènes. Les propriétaires peuvent avoir intérêt à favoriser le développement des recherches académiques sur les gènes brevetés pour récupérer les connaissances qui seront produites par les chercheurs ${ }^{19}$. D'un autre côté, si le brevet divulgue la connaissance sur le gène, il en réserve l'usage au bénéfice exclusif du premier découvreur, ce qui introduit une chaîne de dépendance pour tous les innovateurs ultérieurs. La réservation exclusive de l'usage de la ressource génétique est alors susceptible de réduire les incitations à entreprendre de nouvelles recherches sur les gènes ou à réaliser de nouveaux développements sur les méthodes de tests génétiques.

Les enquêtes réalisées aux États-Unis en Californie et en Pennsylvanie semblent confirmer ce phénomène. Une enquête conduite par Cho, Leonard, Merz en 1999 sur une population de soixante-quatorze laboratoires cliniques montrait que $25 \%$ avaient abandonné le test clinique qu'ils avaient développé en raison de brevets pendants et que $48 \%$ avaient renoncé à développer un test clinique en raison de l'existence de brevets. La Clinical Molecular Genetics Society du Royaume-Uni exprimait ainsi les risques de blocage associés aux brevets sur les gènes : «Chaque nouvelle technologie sera captive d'un brevet qui restreindra la liberté de la recherche et du développement dans ce domaine et qui minera la capacité du secteur public du Royaume-Uni et de ses partenaires de l'industrie biotechnologique à développer un potentiel de recherche et de nouveaux produits. » La tendance des propriétaires de gènes à imposer leur standard de test génétique, à l'instar de Myriad Genetics, réduit la variété des développements technologiques. La réduction du nombre de laboratoires susceptibles 
de réaliser les tests génétiques nuira également à l'accumulation des connaissances sur les gènes qui sont souvent encore très incomplètes, aussi bien pour ce qui concerne leur fonction biologique que les risques associés aux différentes mutations génétiques.

Outre les problèmes liés à la dépendance, il existe une autre difficulté liée à la fragmentation de la connaissance et de la propriété des gènes. Il peut en résulter un effet négatif sur l'innovation, lié au coût nécessaire à la négociation et à la levée de multiples droits de propriété. Ce phénomène pourrait notamment survenir pour des maladies qui relèvent de plusieurs gènes, dès lors qu'il est nécessaire de négocier des droits auprès de multiples propriétaires pour développer un test génétique. Holtzman et Hilgartner (1998) ont relevé un tel cas dans le domaine du test prénatal. Il pourrait également se produire dans le domaine du cancer du côlon dès lors que les principaux gènes sont distribués entre deux centres propriétaires.

\section{L'impact de ces brevets sur l'offre de santé}

Les propriétaires des gènes, particulièrement les sociétés de génomique, utilisent leur position pour construire des marchés réservés des services de santé, en l'occurrence des tests génétiques, et pour capturer l'offre de services qui étaient réalisés jusqu'ici par des laboratoires universitaires ou hospitaliers. Il en résulte une gestion très fermée de la propriété intellectuelle, par le biais de licences exclusives ou asymétriques.

Les licences proposées par Myriad Genetics à des laboratoires hospitaliers ou industriels pour la commercialisation des tests génétiques pour les cancers du sein et du côlon illustrent parfaitement cette asymétrie. Prenons l'exemple du laboratoire clinique de l'université de Pennsylvanie qui avait développé une méthode originale de tests dès la publication des gènes du cancer du sein en 1994 et 1995. En 1997, il réalisait environ cinq cents tests par an qui étaient vendus au prix de revient. Dès que les brevets demandés par Myriad Genetics furent délivrés, en décembre 1997, cette société fit parvenir une lettre à l'université la prévenant qu'elle enfreignait ses droits et lui proposa un accord de licence. La licence proposée ne permettait plus à l'université de rechercher la mutation familiale dans l'ensemble du gène. Cette partie du test, la plus décisive, serait réalisée dans l'usine à tests de Myriad à Salt Lake City, moyennant un prix de 2400 dollars. Elle l'autorisait en revanche à rechercher la mutation, une fois celle-ci connue, parmi les autres membres de la famille - cette partie du test est la plus routinière -, moyennant une redevance de 45 dollars. Les cliniciens essayèrent de négocier une licence plus équilibrée qui leur aurait permis de réaliser le screening complet des gènes, mais Myriad refusa 20 .

Cette gestion très fermée de la propriété industrielle n'est pas spécifique aux gènes du cancer du sein. Dans une enquête publiée en 1999 dans Nature, Schissel, Cho et Merz ont montré que sur un panel de vingt-sept gènes, les quatorze licences qui avaient été conclues à la date de l'enquête étaient toutes des licences exclusives. Notamment, des brevets couvrant les diagnostics génétiques de plusieurs maladies neurologiques avaient tous étés licenciés de manière exclusive à une compagnie de biotechnologie, Athena Diagnostic. Si les sociétés de génomique gèrent leurs brevets de manière restrictive afin de rentabiliser leurs plates-formes de séquençage et de valoriser leurs actifs sur les marchés financiers, certaines institutions médicales adoptent les mêmes pratiques. Ainsi, l'hôpital des Enfants de Miami, qui a isolé et breveté le gène responsable d'une maladie cérébrale d'origine génétique, la maladie de Canavan, est poursuivi en justice par des parents d'enfants malades pour une gestion restrictive de sa propriété : en plus des royalties perçues pour chaque test, l'hôpital a limité le nombre de laboratoires susceptibles de réaliser le test (Chicago Tribune, 22 janvier 2001).

Les cliniciens ont souligné les impacts négatifs de l'émergence de monopoles commerciaux dans le domaine des tests génétiques, notamment : Les risques de concentration de l'offre de soins au bénéfice de quelques centres propriétaires ou licenciés - à l'opposé du libre développement des tests génétiques et d'une offre largement distribuée lorsque l'usage des gènes est libre ;

La fragmentation des services de médecine moléculaire dès lors qu'une seule société ne peut détenir des droits sur tous les gènes ;

L'accumulation de connaissances dans les centres qui réaliseront les analyses les plus importantes et qui collectionneront les mutations et l'étiolement de l'expertise des laboratoires qui ne réaliseront plus que des tests de routine ; 
L'augmentation des prix des tests - le prix des tests du cancer du sein pourrait doubler en France et au Royaume-Uni, où les laboratoires hospitaliers ont développé des méthodes de tests plus légères et moins onéreuses que celle imposée par le propriétaire des gènes ;

Les problèmes de pérennité de l'offre de soins dès lors que la fourniture d'un test sera dépendante d'une société privée qui peut subir les aléas du marché.

Enfin, certains font valoir que ces brevets peuvent empêcher des médecins de pratiquer la médecine - sous peine de devenir des contrefacteurs. Resurgit ici un vieil argument du conflit entre l'éthique médicale et l'attribution de droits exclusifs sur les produits de santé ${ }^{21}$.

\section{Des modèles de production de biens collectifs et de biens publics 22}

La tendance à la privatisation des connaissances, aussi forte soit-elle dans la génomique, ne recouvre pas l'ensemble des modes de production et de distribution des connaissances qui y sont à l'œuvre. Tout d'abord, la communauté scientifique a inventé des systèmes d'invention collective qui reposent sur le partage des connaissances. Ensuite, le mouvement de privatisation, et ses excès, produit ses propres limitations (par exemple, lorsque les grands laboratoires pharmaceutiques décident d'organiser un consortium de recherche qui versera ses résultats dans le domaine public). Enfin, le modèle du bien public ou commun est susceptible de favoriser l'accessibilité des nouveaux produits de santé et de satisfaire aux critères et aux normes de la santé publique (par exemple, le modèle du libre usage des gènes et du libre développement des tests génétiques qui existe dans les laboratoires hospitaliers en France, en Grande-Bretagne, le cas échéant aux États-Unis).

\section{Le modèle du Centre d'étude du polymorphisme humain : un club scientifique ouvert à tous les laboratoires intéressés}

Le CEPH est une fondation de recherche française privée créée par Jean Dausset, le découvreur du système HLA, et Daniel Cohen. Cette organisation non profitable a inventé au début des années quatre-vingt un système original de recherche collective pour cartographier le génome humain. L'objectif du CEPH était de mettre à disposition de la communauté scientifique un pool de familles de référence pour construire une carte génétique. $\mathrm{Au}$ lieu que chaque laboratoire balise le génome sur ses propres familles, le CEPH entendait promouvoir un travail en réseau. Chaque laboratoire qui le souhaitait pouvait utiliser les mêmes échantillons de référence pour tester ses propres sondes, identifier et localiser des marqueurs génétiques. Les données produites par chaque participant étaient centralisées dans une base de données et comparées pour générer une carte commune. Des sous-consortiums furent organisés pour valider les données et produire la carte de chaque chromosome. Une telle organisation collective augmente la croissance cumulative des connaissances : premièrement, il existe un avantage particulier à ce que tout le monde travaille sur les mêmes familles pour baliser le génome : «L'idée était superbe ; travailler sur les mêmes méioses, ça vous donne infiniment plus d'informations pour en faire une carte » (Jean Weissenbach) ; deuxièmement, la centralisation des données pour les comparer et les assembler facilite la production d'une carte pour laquelle il faut positionner les balises les unes par rapport aux autres.

Ce système d'invention collective a produit sa propre « constitution » au cours d'une réunion fondatrice en 1984. Les règles d'adhésion au réseau du CEPH sont les suivantes : tous les laboratoires intéressés peuvent devenir membres du réseau - $C E P H$ investigator ; leur demande d'adhésion est examinée par le comité scientifique du CEPH ; les participants peuvent accéder gratuitement à la collection des familles ; en contrepartie, ils s'engagent à tester les quarante familles sélectionnées avec leur sonde, soit dans leur laboratoire, soit en collaboration. Ils doivent également s'engager à adresser au CEPH leurs résultats, et cela avant publication. Cette mise à disposition des résultats avant publication vise à favoriser les collaborations au sein du réseau. Chaque participant peut bénéficier des résultats des autres membres grâce à une distribution régulière des données à jour. En revanche, le CEPH n'a pas imposé de règles pour l'échange des sondes que possèdent les laboratoires : «Nous laissons les investigateurs décider des conditions de mise à disposition de leurs sondes. » 
Le réseau du CEPH a défini deux cercles de diffusion des données. Tout d'abord, les données circulent au sein du réseau : le CEPH a construit une base de données collaborative - collaborative database - qui contient les données publiées et non publiées. Cette base de données est un bien collectif partagé entre les membres du réseau. Le CEPH a défini un standard pour la mise en forme des données produites - pour cela, il distribue gratuitement un logiciel à tous les participants. Ensuite, il gère une base de données publique, accessible à toute la communauté scientifique - the CEPH public database. Les données collaboratives sont versées dans la base de données publique au plus tard après une période de deux ans : " Après un dépôt de deux ans dans la base collaborative, les données non publiées sont publiées. »

La réunion de la collection de familles n'a pas été sans difficultés. Les fondateurs du CEPH ont négocié au départ avec Ray White qui avait déjà engagé un travail de cartographie du génome humain à l'université d'Utah à partir d'une collection de familles de mormons. Ray White refusa dans un premier temps de fusionner ses familles avec celles de Jean Dausset. Il décida finalement de participer à la recherche collective. $\mathrm{Ce}$ pool de familles américaines et françaises s'est progressivement imposé comme un point de passage obligé pour la cartographie du génome humain. Si la réunion fondatrice du CEPH avait rassemblé treize laboratoires, le réseau s'est rapidement étendu - cinquante l'année suivante, une centaine au début des années quatre-vingt-dix, dont une majorité de laboratoires américains. Cette position privilégiée suscita en 1991 un conflit avec les NIH qui tentèrent de substituer au pool du CEPH une collection de familles de très grandes tailles d'origine vénézuélienne. Cette tentative échoua devant l'opposition des laboratoires collaborateurs qui mirent en avant le travail accumulé pendant huit ans.

Les fondateurs et les membres du réseau du CEPH ont choisi de ne pas prendre de droits de propriété intellectuelle sur les marqueurs qu'ils avaient identifiés : «On a conclu qu'il n'y avait pas de propriété intellectuelle à prendre là-dessus ; ça devait être disponible publiquement » (Jean Weissenbach). De fait, les cartes du génome humain produites par le CEPH puis par le laboratoire du Généthon en France furent largement publiées et sont utilisées par tous les laboratoires de par le monde. Les enjeux de propriété intellec- tuelle s'aiguisèrent avec l'accélération du séquençage au milieu des années quatre-vingtdix.

\section{Les consortiums européens : pools de données et systèmes de propriété collective}

Les programmes de recherche européens de génomique ont également créé des espaces de recherche collective et publique (Cassier, Foray, 2001). Il s'agissait pour les promoteurs du programme de séquençage de la levure en 1989 de construire une organisation de recherche en réseau qui fédère les laboratoires de plusieurs pays européens. Les différents consortiums que nous avons étudiés ont inventé des dispositifs originaux de diffusion et d'appropriation des données de recherche qu'ils ont formalisés sous forme de guidelines.

Les consortiums européens ont tout d'abord inventé des dispositifs de gestion des données qui s'efforcent de concilier protection individuelle des participants, apprentissage collectif au sein du réseau et diffusion dans le domaine public. Les règles de diffusion concentrique des données établissent trois degrés d'accessibilité des données, en fonction du temps : pendant une période de un ou trois mois, les participants bénéficient d'un droit d'usage prioritaire sur les données qu'ils ont produites ; lors d'une deuxième période, les données sont partagées entre les membres du consortium ; puis elles sont versées dans le domaine public. La négociation entre les participants porte sur les délais de passage d'un cercle à un autre, dès lors que des industriels souhaitent augmenter la durée de réservation des données dans l'espace individuel ou collectif ou que des universitaires souhaitent publier au plus tôt. Ces consortiums parviennent à générer des pools de connaissances, plus ou moins étendus, sous forme de collections de données ou de matériels biologiques, qui favorisent la production collective.

Ces consortiums ont expérimenté différents régimes de répartition de la propriété intellectuelle sur les résultats de recherche, depuis des systèmes de propriété disjointe, notamment dans des consortiums qui réunissent des industriels concurrents, jusqu'à des systèmes de propriété collective, notamment dans des consortiums à dominante académique. Les formules de propriété collective ont un intérêt particulier pour la 
gestion des connaissances. Parmi les huit consortiums étudiés, nous en avons identifié trois qui avaient élaboré un régime de propriété collective. Dans deux cas, les résultats sont le produit d'une recherche profondément collective - les données résultent de la combinaison de multiples tests et expertises réalisés par plusieurs laboratoires - , si bien qu'il n'est pas raisonnable d'attribuer telle ou telle donnée à l'un ou l'autre des participants. Dans le cas restant, les participants ne disposent pas d'un outil de partage de la propriété, c'est-àdire d'une carte génétique ou physique, pour répartir des morceaux de chromosome bien identifiés. Dans ces trois cas, les participants s'accordent sur la définition d'une propriété collective qui sera gérée par une institution ad hoc - un Charity Trust pour le consortium EUROFAN - ou par l'un des participants - l'Institut Pasteur pour le consortium sur la listeria.

Ces formules de propriété collective favorisent la constitution d'une base de connaissances ayant une certaine cohérence, à l'opposé de la fragmentation de la connaissance génomique relevée par Heller et Eisenberg. Les problèmes de recouvrements de propriété et les risques de conflits sont résolus en amont : une seule institution est habilitée à prendre des droits sur les résultats du consortium. Les utilisateurs n'auront qu'un seul interlocuteur pour négocier des droits d'accès aux données. Une telle solution est de nature à prévenir les situations « d'anticommuns » dans la mesure où l'on évite le morcellement de la propriété et où l'on diminue les coûts de transaction pour accéder aux connaissances. Toutefois, une telle appropriation collective ne résout pas tous les problèmes d'accès : elle pourrait être confisquée par un club qui définirait des licences exclusives ou restrictives pour l'usage de ces données.

\section{Le consortium SNP : quand le secteur privé instaure un système de production de biens publics}

En 1999, dix grands laboratoires pharmaceutiques mondiaux s'associèrent à des centres universitaires et au Wellcome Trust pour produire une carte de marqueurs génétiques qui devait être mise à la disposition immédiate de la communauté scientifique. Cette organisation de recherche collective entend produire rapidement une ressource stratégique pour les laboratoires pharmaceutiques - un catalogue des variations génétiques qui sera utilisé pour concevoir de nouveaux diagnostics et des médicaments adaptés à des groupes spécifiques de patients - et prendre de vitesse les sociétés de génomique, Celera et Incyte Pharmaceuticals, qui se sont engagées dans la constitution de bases de données privatives sur les mêmes marqueurs.

Le projet initial du consortium n'avait pas tranché en faveur d'un accès réservé aux laboratoires pharmaceutiques membres du consortium ou d'un accès ouvert à tous les utilisateurs. Finalement, les différents participants, industriels, universités et fondation, s'accordèrent pour créer une base de données ouverte à tous. Les justifications avancées par l'un des participants industriels pour placer ces données dans le domaine public sont de deux types. Premièrement, le versement des marqueurs SNP dans des bases de données privées augmenterait le coût d'usage des données pour les laboratoires pharmaceutiques. Deuxièmement, le versement des SNP dans le domaine public favorisera le processus cumulatif de création de connaissances : «Cela accroît la base de connaissances publiques et tout le monde en bénéficie » (Paul Spence, Pharmacia Research). Les intérêts des laboratoires académiques et des firmes pharmaceutiques convergent pour augmenter la base de connaissances publique et s'opposent aux intérêts des sociétés privées de génomique. Tandis que l'information sur les SNP a une valeur commerciale directe pour des sociétés comme Celera ou Incyte, elle représente un coût pour la recherche industrielle des laboratoires pharmaceutiques. Ces derniers entendent bénéficier des effets indirects et diffus de la recherche publique tandis que Celera ou Incyte espèrent un revenu immédiat de la réservation des marqueurs.

La stratégie du consortium SNP pour garantir l'accessibilité des résultats de la recherche ne repose pas seulement sur la publication immédiate des marqueurs. Le consortium utilise les brevets, à des fins défensives pour préserver l'accessibilité des SNP : «Nous ne voulons pas seulement publier l'information, mais avant de publier, nous utilisons le système des brevets contre luimême afin de préserver l'accessibilité des données » (Arthur Holden, directeur du consortium). Pour cela, le consortium utilise la procédure des brevets à titre provisoire - provisional patents qu'il abandonne une fois le marqueur publié. Ce faisant, la date de chaque marqueur SNP est enre- 
gistrée à l'USPTO et peut servir de preuve d'antériorité face à des revendications de propriété 23 .

\section{Le « modèle du commun » dans l'usage des gènes de susceptibilité}

Tandis que des sociétés de génomique brevettent les gènes et gèrent leur propriété de manière restrictive pour construire des monopoles sur les tests génétiques, certains découvreurs de gènes ont adopté un régime de licence très ouvert afin de favoriser l'accessibilité des tests génétiques. Il s'agit par exemple de la politique de l'université du Michigan, qui, arrivée la première dans la course au gène de la mucoviscidose, décida de déposer un brevet et d'accorder des licences non exclusives et à très bas prix : " Il fut décidé d'interdire les licences exclusives et de demander une redevance symbolique de seulement deux dollars par test. » Il s'agit également de la pratique des cliniciens et des chercheurs qui utilisent librement les gènes publiés pour développer et fournir des tests génétiques aux patients. Dans ce modèle, les gènes sont un bien commun de la recherche et de l'innovation médicale.

\section{Conclusion}

De manière plus prospective, on peut envisager plusieurs solutions pour parvenir à un meilleur équilibre entre bien privé, bien collectif et bien public. Ce rééquilibrage étant doublement justifié, pour préserver l'ouverture du système de recherche et pour satisfaire le bien public en matière de santé.

On peut tout d'abord envisager une réorientation du système des brevets en excluant les gènes de la brevetabilité. C'est la position de plusieurs associations de scientifiques, de cliniciens ou de malades (par exemple, le Position Statement on Gene Patents and Accessibility of Gene Testing de l'American College of Medical Genetics, août 1999). Dans ce cadre, la séquence génétique, qu'elle soit partielle ou complète, brute ou interprétée, ne pourrait être revendiquée par des brevets. Seuls les procédés d'application des gènes, effectivement démontrés et bien délimités pour éviter des brevets trop larges, ou encore des produits dérivés de la connaissance de la séquence - molécules pharmaceutiques - pourraient être brevetés ${ }^{24}$. Les brevets accordés auraient un moindre pouvoir de monopole que ceux qui couvrent à la fois le gène et ses utilisa- tions. Ils auraient l'avantage de laisser les gènes en accès libre, chaque utilisateur ayant la possibilité de concevoir de nouvelles applications et de nouveaux produits sans être dans l'obligation de s'acquitter d'une rente auprès du découvreur du gène. Cette disposition est adaptée à la polysémie des gènes, qui sont susceptibles d'avoir plusieurs fonctions biologiques selon l'environnement.

La diffusion libre des séquences génétiques pourrait aussi s'inspirer du modèle de la Licence Publique Générale, qui organise la libre circulation et le libre usage des connaissances et en garantit la non-appropriation.

Les institutions publiques de recherche pourraient également, à l'instar du consortium SNP ou de Louis Pasteur en son temps, utiliser le système des brevets pour préserver l'accessibilité de leurs travaux. Il s'agirait de verser systématiquement dans le domaine public les brevets déposés une fois la séquence publiée. Elles pourraient également constituer des pools de brevets, pour éviter la fragmentation des connaissances, et adopter des politiques de licences non-exclusives, pour prévenir l'émergence de monopoles sur le terrain de la santé. Les consortiums ou les organisations en réseau représentent également des solutions locales à la privatisation excessive des connaissances, pour peu que des acteurs veillent à leur ouverture minimale.

Il s'agit enfin de définir un nouveau compromis entre la propriété industrielle et la santé publique en définissant des régimes de licences très ouverts pour garantir l'accessibilité des innovations médicales - c'est la position de l'American College of Medical Genetics -, ou encore des licences conditionnelles qui lient une large distribution des innovations au respect de bonnes pratiques médicales - c'est le modèle de la licence de la Cancer Research Campaign pour le gène BRCA2 -, ou encore d'utiliser les licences d'office dans l'intérêt de la santé publique - c'est une solution évoquée par la Clinical Molecular Genetics Society du Royaume-Uni ou par des cliniciens français face aux brevets de Myriad Genetics sur les gènes du cancer du sein.

Ces différents mécanismes institutionnels sont susceptibles d'être combinés pour parvenir à des normes d'appropriation acceptables. Si l'intégration de la science et du marché est particulièrement avancée dans le domaine de la génomique, les enjeux de santé publique l'accessibilité et la diffusion des innovations médicales - poussent à définir des compromis 
originaux entre ce qui relève des biens privés et des biens publics. La négociation de compromis entre la logique industrielle et la logique de santé publique suppose l'intervention de la représentation politique 25 et la participation d'acteurs variés, incluant notamment les organisations nongouvernementales (ONG), associations de scientifiques, de médecins et de malades, qui réfléchissent sur la propriété industrielle et la santé.

\section{Notes}

1. Le patrimoine collectif des villageois était considéré jusqu'alors comme un bien inaliénable, utilisable par tous les membres de la communauté, et qui s'étendait également aux générations futures. Son partage marque l'avancée de la propriété exclusive et la désagrégation ou le recul de la propriété communautaire sur la terre. Cf. K. Polanyi, La grande transformation, p. 238.

\section{La réaction à la baisse des} marchés financiers à la déclaration du président Clinton et du premier ministre britannique Tony Blair du 14 mars 2000, qui invitaient les acteurs publics et privés de la génomique à placer les résultats du décryptage du génome dans le domaine public, est symptomatique de cette nouvelle intégration.

\section{Le « groupe du phage»} constitué autour de Max Delbrück dans les années quarante aux États-Unis était également un réseau informel dans lequel circulaient des objets techniques mis en commun : « De plus, les participants apprenaient à utiliser les mêmes objets d'étude et les mêmes techniques, ce qui contribuait en retour à donner à l'approche initiée par M. Delbrück une influence croissante » (M. Morange, 1994).

\section{Genset et Myriad Genetics} utilisent des contrats d'accès réservé aux collections de données génétiques et médicales, des accords de transfert de matériel qui fixent la répartition de la propriété entre les contributeurs, de nombreux contrats de recherche avec les universités et les laboratoires pharmaceutiques, le secret commercial pour la protection de bases de données, des brevets sur des gènes associés à des maladies ou sur des procédés de fabrication synthétique des gènes, etc. Les contrats permettant de couvrir des connaissances très hétérogènes et de compléter ainsi les brevets.

5. On sait que les familles de l'Utah présentent un avantage particulier dans la recherche des gènes de prédisposition et qu'elles ont attiré de nombreux chercheurs et sociétés. En vertu des accords conclus avec l'université d'Utah, Myriad Genetics détient un droit d'exploitation exclusive d'une base de données généalogiques qui couvre 1,25 millions d'individus.

Cette base de données a été développée à l'origine par le fondateur de la start-up, Mark Skolnick.

6. « Nous pensons que certaines caractéristiques uniques de la population islandaise, associées à nos capacités avancées en bioinformatique et en génotypage, donnent à DeCode un avantage compétitif créateur de valeur pour notre société et nos partenaires » (DeCode).

7. Une disposition du projet de loi initial formulait explicitement la possibilité de restriction des investigations scientifiques sur la base de données : «Le comité peut accorder aux chercheurs mentionnés ci-dessus l'accès à la base de données à des fins de recherche, à condition que la recherche ne soit pas susceptible, du point de vue du comité, d'avoir un effet négatif sur l'intérêt commercial du licencié » (article 9). La loi finalement adoptée ne contient plus cette disposition, mais reste muette sur les possibilités d'accès des chercheurs extérieurs. En 1999, le Ministre de la Santé islandais réitérait ces restrictions : «Les chercheurs extérieurs pourront accéder à la base de données pour autant que des intérêts commerciaux ne sont pas en jeu. » De très nombreux scientifiques se sont élevés contre cette restriction (cf. H.T. Greely, M.C. King, " Letter to the Government of Iceland », 1998). Réagissant à la licence accordée à DeCode, The World Medical Association s'est aussi prononcée pour un accès libre à toute donnée scientifique (16 avril 1999).

8. Tout patient ne déclarant pas explicitement son opposition sera considéré comme ayant donné son accord.

9. «Utility examination guidelines », USPTO, Federal Register, vol. 66, $\mathrm{n}^{\circ} 4$, vendredi 5 janvier 2001. Ces guidelines sont précédés d'une discussion des commentaires publics qui ont été recueillis lors de la phase d'élaboration de ces règles. Ces commentaires émanaient de trente-cinq individus et de dix-sept organisations.

10. Ainsi, un des pionniers de la recherche génomique, Walter 
Gilbert, qui cherchait à la fin des années quatre-vingt à réunir des fonds pour créer une société de commercialisation de

l'information génétique, envisageait en 1987 de protéger cette information par le copyright Cf Cook-Degan, The Gene Wars, 1994, p. 309.

11. Ce sont en effet les spécialistes de la propriété industrielle et les innovateurs qui ont traduit les critères de brevetabilité - invention, nouveauté, activité inventive, utilité ou application industrielle - en règles pratiques applicables aux objets de la génomique. En étendant le champ de la brevetabilité à de nouvelles classes d'objets, par exemple aux séquences partielles ou complètes de gènes, généralement très en amont des applications médicales, ces décisions influent sur le processus d'innovation et sur l'allocation des ressources.

Autrement dit, brevette-t-on les bons objets et récompense-t-on les bons acteurs?

12. La directive européenne sur la protection juridique des inventions biotechnologiques en 1998 a retenu les mêmes critères d'interprétation : «Un élément isolé du corps humain ou autrement produit par un procédé technique, y compris la séquence ou la séquence partielle d'un gène, peut constituer une invention brevetable, même si la structure de cet élément est identique à celle d'un élément naturel. »

13. « Il serait ironique et fâcheux que le système des brevets récompense la routine et décourage l'innovation » (Hugo Statement on the Patenting of DNA Sequences, 1995).

14. Dans le domaine du cancer du sein, Myriad Genetics a utilisé les brevets sur les gènes pour construire un marché réservé des tests génétiques. Les universités américaines ont breveté ces gènes pour valoriser leurs recherches et pour limiter le monopole de Myriad Genetics. Une fondation britannique d'aide à la recherche contre le cancer, The Cancer Research Campaign, a breveté le gène qu'elle avait découvert pour préserver son accessibilité et afin de contrôler l'usage médical qui pourrait en être fait (Cassier, Gaudillière, 2000).

\section{5. «Quand un brevet}

revendiquant un nouveau composé chimique est accordé, le titulaire du brevet a le droit d'exclure les autres de la fabrication, de l'utilisation, de la mise en vente, de la vente, de l'importation de ce composé pour une période donnée. Le titulaire du brevet est tenu de divulguer seulement une utilité, c'est-à-dire d'instruire les autres sur une seule manière d'utiliser l'invention » (Utility Examination Guidelines, USPTO, op. cit.).

16. « Du fait qu'il n'y a qu'un seul génome humain, en l'aliénant, vous établissez inévitablement une position de monopole et cela au détriment de la biologie » (John Sulston, directeur du Sanger Center).

17. On estime que pour de nombreuses applications médicales, c'est la protéine et non la séquence génétique qui aura une valeur d'usage intéressante. Dans ce cas, l'appropriation de la séquence permettra d'exercer un contrôle sur le produit lié, la protéine.

18. Lors des débats au parlement européen sur la directive sur la protection juridique des inventions biotechnologiques, le rapporteur de la directive soulignait l'enjeu de l'inclusion des séquences génétiques dans les brevets : «Une protection par brevets ne peut être efficace que si elle porte également sur la substance qui contient les informations nécessaires. » Il illustrait son propos par la découverte d'un contrepoison dans un champignon : « Si la protection par brevets ne portait pas sur cette substance, elle serait dénuée de valeur, car ce poison serait accessible à n'importe qui sous une autre forme qu'un médicament. C'est pourquoi cette protection de la substance est capitale et c'est pourquoi le projet que nous présentons ne rompt absolument pas avec l'actuel droit des brevets. » (Séance du $1^{\mathrm{er}}$ mars 1995.)

19. Par exemple, en janvier 2000 , Myriad Genetics signait un accord avec le National Cancer Institute pour réduire fortement le coût du séquençage des gènes du cancer du sein à usage de recherche. Toutefois, les propriétaires de gènes peuvent aussi utiliser leur position pour définir des contrats très déséquilibrés sur les connaissances qui seront produites par les chercheurs ou même pour limiter l'usage de leur matériel ou procédé. Ainsi, Myriad Genetics at-elle interdit à l'université de Pennsylvanie de réaliser des tests sur les gènes du cancer du sein pour un consortium de recherche, en considérant qu'il s'agissait d'un service commercial.

20. Les licences proposées par Myriad aux centres anticancéreux français en décembre 1998, qui anticipaient la délivrance prochaine des brevets européens qu'elle avait demandés, reposent sur le même partage du travail.

21. «La ruée au brevetage des découvertes génétiques entre en conflit avec la position morale de l'American Medical Association, en vigueur depuis plus de cent cinquante ans, parce qu'elle donne le droit à des médecins de restreindre et de tirer directement profit de la pratique de la médecine par d'autres praticiens. » («Statement to the Subcommittee on Courts and

Intellectual Property of the

Committee on the Judiciary U.S. 
House of Representatives Oversights Hearings on Gene Patents and Other Genomic Inventions », July 13, 2000, by J.F. Merz, University of Pennsylvania. )

22. Nous différencions les biens collectifs, qui sont accessibles aux membres d'un réseau ou d'une communauté scientifique ou professionnelle, et les biens publics, qui sont accessibles à tout utilisateur potentiel. Les deux ne se recouvrent pas nécessairement. Dans certains cas de figure, les biens collectifs sont à la disposition d'un club fermé, et ils excluent les autres utilisateurs.
23. Louis Pasteur avait également utilisé le dépôt de brevet pour empêcher la confiscation de ses inventions. Il déposa ainsi un brevet sur un procédé de fabrication du vinaigre qu'il mit aussitôt dans le domaine public. Il établissait ainsi son antériorité et favorisait la diffusion de ce procédé dans le tissu industriel.

\section{L'European Campaign on} Biotechnology Patents propose le partage suivant entre ce qui est brevetable et ce qui ne doit pas l'être : « Les signataires pensent que l'étendue d'un brevet doit être limité aux usages factuellement démontrés et que, de plus, la séquence d'un gène ne peut en tant que telle faire l'objet d'un brevet. Il devrait aussi être possible que les différent produits ou thérapies faisant usage de la séquence génétique soient couverts par des brevets séparés. » (Protest against Patent on « breast cancer gene » (BRCA1) - Objection to third Party under Article 115 of the European Patent Convention.)

25. Martin Bobrow and Sandy Thomas, 2001, avancent l'idée d'un déficit d'implication de la représentation politique dans l'élaboration des politiques de propriété intellectuelle touchant au génome.

\section{Références}

Bobrow, M. ; ThOMAs. 2001.

«Intellectual property rights in a

Genetic Age », Colloque de l'UNESCO sur Éthique, propriété intellectuelle et génomique, Paris, 29 janvier-1 ${ }^{\text {er }}$ février.

CAssier, M. 2000. " Patent and public health : genome patents nowadays and pharmaceutical patents in the 19th : a parallel », International Conference Technological Policy and Innovation : Economical and Historical Perspectives, Paris, 2022 novembre.

-. ; GAUdilliÈre, J.-P. 2000, Les Relations entre science, médecine et marché dans le domaine du génome : pratiques

d'appropriation et pistes pour de nouvelles régulations, rapport pour le programme Génome du CNRS, $68 \mathrm{p}$.

-. ; Foray, D. 2001. « Public knowledge, private property and economics of high-tech consortia : case studies in bio-medical research », dans Bellon, B. (dir. publ.), Industrial Cooperation,
Diversity and Synthesis, London, Edward Elgar.

Cohen, D. 1992. Les Gènes de l'espoir, Paris, Robert Laffont.

CoOK-Degan, R. 1994. The Gene Wars, Science, Politics and the Human Genome, New York, London, WW Norton \& Company.

EISENBERG, R. 1996. Intellectual Property at the Public-Private Divide : The Case of Large-Scale cDNA Sequencing, 557-573.

Heller, M. ; EISEnBerg, R. 1998. «Can patents deter innovation? The anticommons in biomedical research », Science, vol. 280, 1 .

HoltZMan, N. A. ;

HILGARTNER, S. 1998. « State of the art of genetic testing in the United States : survey of biotechnology companies and nonprofit clinical laboratories and interviews of selected organizations », dans Holtzman, N.A. ; Watson, S. (dir. publ.), Promoting Safe and Effective Genetic Testing in the United
States, Final Report of the Task Force on Genetic Testing, Baltimore, The Johns Hopkins University Press.

KHOLER, R. 1994. The Lords of the Fly, Chicago, University Chicago Press.

Morange, M. 1994. Histoire de la biologie moléculaire, Paris,

La Découverte.

OCDE, 1995. Le Grand Programme sur le génome humain, Paris.

POLANYI, K. 1983 (1944). La Grande Transformation, Aux origines politiques et économiques de notre temps, Paris, Gallimard.

RIFKIN, J. 1998. The Biotech Century : Harnessing the Gene and Remaking the World, New York, P. Tarcher/ G.P. Putman'Sons.

SCHISSEL, A. ; MERZ, J.F. ; CHO, M.K. 1999. « Survey confirms fears about licensing of genetic tests », Nature, volume $402, n^{\circ} 6758$. 